\title{
5. O problema das admissões em universidades
}

\author{
Aline Guarnieri Gubitoso \\ Vinicius Cifú Lopes
}

\section{SciELO Books / SciELO Livros / SciELO Libros}

GUBITOSO, G., and LOPES, V. C. O problema das admissões em universidades. In: Alocações, estabilidade e otimização: uma introdução passo a passo [online]. São Bernardo do Campo, SP: Editora UFABC, 2017, pp. 91-105. ISBN: 978-85-6857-682-3. https://doi.org/10.7476/9788568576823.0006.

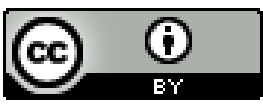

All the contents of this work, except where otherwise noted, is licensed under a Creative Commons Attribution 4.0 International license.

Todo o conteúdo deste trabalho, exceto quando houver ressalva, é publicado sob a licença Creative Commons Atribição $\underline{4.0}$.

Todo el contenido de esta obra, excepto donde se indique lo contrario, está bajo licencia de la licencia $\underline{\text { Creative }}$ Commons Reconocimento 4.0. 


\section{5 \\ O problema das admissões em universidades}

A discussão do problema do casamento não abrange a maioria dos problemas enfrentados na economia contemporânea, mesmo aqueles sem mecanismo de preços, devido ao fato de que nesses mercados do mundo real não são estruturadas somente alocações entre dois conjuntos de agentes que se combinam em pares.

Assim, consideraremos, agora, os dilemas de situações mais complexas, como o intitulado problema das admissões em universidades, em que alocamos a uma única universidade mais do que um único estudante, pois cada curso universitário busca obter uma determinada quantidade máxima de alunos matriculados.

Dessa forma, temos dois grupos, universidades e estudantes, de modo que os estudantes buscam ser admitidos nas universidades, enquanto as universidades procuram admitir uma certa quantidade de estudantes (na literatura: "quota”), escolhidos a partir de uma avaliação das qualificações destas inscrições. Um exemplo de elemento considerado para a ordenação das preferências pode ser a nota em uma prova, de modo que os estudantes escolhidos são aqueles com as maiores notas (assumindo que não há nenhum aluno com notas iguais, ou que há critérios de desempate).

Ademais, semelhantemente ao problema do casamento, o conceito de estabilidade, nessas situações, diz respeito à inexistência de bloqueios, ou seja, não há um curso e um estudante que não 
estejam alocados entre si, ainda que assim o preferissem, ou um estudante ou universidade que esteja alocado com um membro do grupo oposto considerado inaceitável. Logo, uma alocação é estável se não é bloqueada por nenhum agente individual (estudante ou universidade) ou por qualquer "par" de universidade e estudante.

Consequentemente, se não há inscrições suficientes de estudantes aceitáveis, qualquer universidade pode escolher manter quaisquer vagas sem serem ocupadas, assim como se qualquer estudante não conseguir ser aceito em nenhuma de suas opções aceitáveis de universidade ou se desejar não entrar em nenhuma universidade, ele pode permanecer sem ser alocado a uma universidade. (Essas considerações podem não ser válidas, ou ser limitadas, por normatizações públicas ou internas.)

\subsection{Exemplo com Gale-Shapley}

Para expor o raciocínio pelo qual se desenvolve essa alocação por Gale-Shapley, segue uma demonstração do processo. A título de exemplo, tomamos o universo de 5 estudantes e 2 universidades, cada uma com uma quota de 2 vagas (em um único curso ou ciclo básico), sendo eles: Victor $(V)$; Wilson $(W)$; Xavier $(X)$; Yuri $(Y)$; Zé $(Z)$; Universidade Alfa $(A)$; Universidade Beta $(B)$.

Primeiramente, os estudantes ordenam as universidades segundo a ordem de suas preferências (podendo omitir aquelas em que não desejam estudar em hipótese alguma) e inscrevem-se em suas primeiras opções.

Em seguida, as inscrições são enviadas às universidades, que, por sua vez, listam as inscrições recebidas em uma ordem de preferência, podendo rejeitar as inscrições que não aceitam de forma alguma.

Para nosso exemplo, temos as seguintes listas de preferência: 
Victor (V): $\quad A>B \quad$ Alfa (A) (2 vagas): $\quad V>X>Z>W$

Wilson $(W): \quad A>B \quad$ Beta $(B)$ (2 vagas): $X>Y>W>Z$

Xavier $(X): \quad B>A$

Yuri $(Y): \quad B>A$

Zé $(Z): \quad B>A$

No primeiro passo, como $q=2$ em ambas as universidades, Alfa admite Victor e Wilson, enquanto que Beta admite Xavier e Yuri, mas rejeita Zé:

$\begin{array}{llll}\text { Victor }(V): & A>B & \text { Alfa }(A) \text { (2 vagas): } & V>X>Z>W \\ \text { Wilson }(W): & A>B & \text { Beta }(B) \text { (2 vagas): } & X>Y>W>Z \\ \text { Xavier }(X): & B>A & & \\ \text { Yuri }(Y): & B>A & \\ \text { Zé }(Z): & \hat{B}>A & \end{array}$

Os estudantes rejeitados enviam sua inscrição à sua segunda universidade escolhida e mais uma vez cada universidade escolhe as melhores $q$ inscrições dentre as novas inscrições que recebe e aquelas que já estavam na turma preliminar, formando, então, uma nova turma e rejeitando o resto. $\mathrm{O}$ processo termina quando todo estudante está ou em uma turma preliminar ou foi rejeitado por todas as universidades que deseja. Neste ponto, todas as universidades admitem todos os alunos em suas turmas.

Como Zé foi rejeitado, ele envia sua inscrição para Alfa, que o prefere a Wilson. Na terceira rodada, Wilson envia sua inscrição a Beta, mas esta também o rejeita, porque já completou sua quota com Xavier e Yuri, que prefere a ele.

Por fim, não há mais envio de inscrições, de forma que o processo termina com as admissões de Victor e Zé na universidade Alfa, enquanto que a universidade Beta também completa suas vagas ao admitir Xavier e Yuri.

Neste exemplo, nenhuma universidade permaneceu com vagas ociosas, mas isso é possível, tal qual no problema do casamento com poligamia. 


\subsection{Incertezas na prática descentralizada}

Em um mercado sem um sistema de seleção centralizado, como o Sisu, no Brasil, a quota de vagas de uma universidade pode não ser satisfeita, mesmo que a universidade aprove a mesma quantidade $q$ de inscrições, pois não há como assumir que todos os alunos que são admitidos aceitarão a oferta; de fato, é incerto se um estudante enviou suas inscrições simultaneamente a outras universidades, como ele ordenou as universidades de sua preferência e se outras universidades também irão aceitá-lo, situações essas que poderiam afetar as escolhas do candidato.

Por outro lado, para o estudante, o mesmo processo também é incerto devido à possibilidade de ele ser classificado na lista de espera de uma universidade pouco depois do último estudante contemplado dentro da quota desta universidade, o que significa que, mesmo ele não sendo admitido, ele pode vir a ser futuramente se ocorrer uma desistência.

Em consequência de ambas as perspectivas, o cenário das admissões torna-se problemático: uma pessoa admitida em uma universidade, mas também na lista de espera de outra, que prefere, pode matricular-se na universidade em que é admitido e desistir dela se for chamado pela outra, o que introduz problemas quanto à vaga dele, agora disponível na primeira universidade.

Na Seção 5.4, mesmo com sistemas centralizados, conheceremos problemas semelhantes de colapso e congestão do mercado, um fenômeno em que os agentes participantes não conseguem conhecer uns aos outros e interagir, apesar de sua disponibilidade.

\subsection{Semelhança e distinção com o problema do casamento}

A admissão de mais de um aluno em cada universidade pode ser vista como a escolha dos homens por mais de uma mulher (poligamia), através de um mecanismo em que cada universidade reparte sua capacidade de admissão em "vagas" assim como vimos, na Seção 2.3, os homens fazerem para cada uma de suas mulheres. 
Assim, ao invés de pensar na universidade com capacidade para ingressantes, podemos considerar suas vagas $A_{1}, A_{2}, \ldots, A_{n}$ como diferentes agentes, mas com preferências idênticas às de $A$. Simultaneamente, substituímos $A$ nas listas de preferências dos estudantes pelas vagas $A_{1}, A_{2}, \ldots, A_{n}$, estipulando que eles preferem $A_{1}>A_{2}>\ldots>A_{n}$ para uniformizar a escolha entre vagas equivalentes.

Esse mecanismo foi, de fato, utilizado por Gale e Sotomayor em um estudo teórico (ROTH, 1985, p. 282): esses autores compararam o problema de admissão e o problema do casamento formulado a partir dele, verificando que os emparelhamentos possíveis em um cenário correspondem aos emparelhamentos possíveis no outro e, também, que existe essa correspondência entre os emparelhamentos estáveis de um e do outro.

Roth destacou que ainda existem os ótimos para universidades e para estudantes, como Gale e Shapley indicaram (ROTH; SOTOMAYOR, 1990, cor. 5.9 a respeito de hospitais e médicos residentes).

$\rightarrow$ Propriedade: Gale, Sotomayor e Roth também notaram que, em um mesmo cenário de listas de preferência estrita, todas as possíveis alocações estáveis compartilham as seguintes características:

- Toda universidade preenche sempre o mesmo número de vagas.

- Toda universidade que não completa suas vagas obtém sempre os mesmos estudantes.

- Os estudantes não alocados, se houver, são sempre os mesmos.

Referências: Roth; Sotomayor (1989, teos. 1 e 2; 1990, teos. 5.12 e 5.13) e Gusfield; Irving (1989, teo. 1.6.3).

Entretanto, como o próprio título de Roth (1985) declara, “o problema das admissões em universidades não é equivalente ao problema do casamento". A explicação para isso requer um conceito adicional, o de "otimalidade fraca de Pareto":

$\rightarrow$ Definição: Uma alocação é ótima no sentido fraco de Pareto para um grupo de agentes se não existe outra alocação, seja 
estável ou instável, em que todos os membros desse grupo tenham alocação estritamente melhor.

Na Seção 1.3, explicamos que o emparelhamento monogâmico conduzido pelo algoritmo de Gale-Shapley com propostas feitas pelos homens é ótimo para os homens, dentre os emparelhamentos estáveis, mas não o comparamos imediatamente com todos os possíveis emparelhamentos, inclusive os instáveis. Ainda assim, é possível expandir o raciocínio para mostrar que o resultado de Gale-Shapley é ótimo no sentido fraco de Pareto, ou seja, mesmo nessa comparação mais geral, em qualquer outro emparelhamento algum homem não melhora sua associação. (ROTH; SOTOMAYOR, 1990, teo. 2.27)

Ao desmembrar as universidades em suas vagas, tratamos cada vaga como um agente diferente. Portanto, ao aplicarmos Gale-Shapley, a vaga será atribuída a um estudante e, em qualquer tentativa de substituí-lo por outro estritamente melhor, outra vaga terá que abrigar um estudante de desempenho inferior ou, pelo menos, continuar com o que tem.

Contudo, a universidade ainda pode ter interesse nessa troca, caso não se importe com qual estudante preenche qual vaga e, sim, com a turma ingressante formada como um todo. Roth concluiu que o algoritmo Gale-Shapley produz uma alocação estável entre universidades e estudantes, a melhor possível (dentre as estáveis) do ponto de vista do grupo que faz as propostas, mas não necessariamente ótima no sentido fraco de Pareto. Para vermos como isso ocorre, precisamos esclarecer como as universidades podem comparar turmas:

$\rightarrow$ Definição: Universidades têm preferências responsivas quando, dentre duas turmas que diferem somente pela substituição de um aluno por outro, preferem a turma que contém o melhor desses dois alunos. (ROTH, 1985, p. 282; ROTH, SOTOMAYOR, 1990, p. 128) 
A fim de exemplificar essa situação, tomamos o exemplo de Roth (1985, p. 283), em que temos um universo de universidades (com a quota de cada uma entre parênteses) e estudantes, sendo eles: Alfa $(A)$ (2 vagas); Best $(B)$ (1 vaga); Century $(C)$ (1 vaga); Victor $(V)$; Wilson $(W)$; Xavier $(X)$; Yuri $(Y)$, com as seguintes listas de preferência:

Victor $(V): \quad C>A>B$ Alfa (A) (2): $\quad V>W>X>Y$

Wilson $(W): \quad B>A>C$ Beta $(B)(1): \quad V>W>X>Y$

Xavier $(X): \quad A>C>B$ Century (C) (1): $X>V>W>Y$

Yuri (Y): $\quad A>B>C$

Logo, a partir destas preferências, obtemos a seguinte alocação por Gale-Shapley quando as universidades fazem as propostas:

$$
\begin{array}{llll}
A(2): & V>W>X>Y & V: & C>A>B \\
B(1): & V>W>X>Y & W: & B>A>C \\
C(1): & X>V>W>Y & X: & A>C>B \\
& & Y: & A>B>C
\end{array}
$$

Esse resultado é considerado ótimo (dentre os estáveis) para as universidades. Vemos que $A$ está emparelhada com suas $3^{\text {a }}$ e $4^{\mathrm{a}}$ melhores inscrições, $B$ e $C$ estão cada uma com sua $2^{\mathrm{a}}$ melhor inscrição, ao passo que os estudantes estão todos com sua $1^{a}$ opção de universidade. Em razão disso, resultaria o mesmo se os estudantes fizessem as propostas, e concluímos que se trata da única alocação estável.

Contudo, podemos, manualmente, formar uma nova alocação em que temos:

$$
\begin{array}{llll}
A(2): & V>W>X>Y & V: & C>A>B \\
B(1): & V>W>X>Y & W: & B>A>C \\
C(1): & X>V>W>Y & X: & A>C>B \\
& & Y: & A>B>C
\end{array}
$$


Dessa forma, $B$ e $C$ obtiveram um resultado melhor ao estarem cada uma emparelhada com o estudante que era sua $1^{\mathrm{a}}$ escolha, enquanto que $A$ também melhora, uma vez que, agora, substituiu o $3^{\circ}$ estudante pelo $2^{\circ}$, embora mantenha o $4^{\circ}$. (Como vimos no capítulo anterior, com Roth, 1985 , p. 286 , note que $A$ poderia manipular o resultado e obter essa alocação como proponente, publicando a lista falsa de preferência $W>Y>V>X$.)

Cabe citar um resultado de Roth; Sotomayor (1989, p. 567; 1990, teo. 5.26): com preferências responsivas e estritas entre estudantes, uma universidade pode comparar estritamente as turmas que obteria em diferentes alocações estáveis.

No mundo real, universidades privilegiam notas como critério de admissão, mas podem desejar grupos mais heterogêneos de estudantes, com diversidade de gênero, classes sociais, origem geográfica e internacionalização.

\section{Exercícios}

1) Confira que o algoritmo Gale-Shapley produz o resultado indicado conforme as listas de preferência dadas, tanto com universidades propondo, como com estudantes propondo.

2) Verifique que o emparelhamento alternativo, apresentado por Roth, é instável.

Resposta: $O$ par $(A, X)$ bloqueia a alocação, porque $X>_{A} Y$ e $A>{ }_{X} C$. Para satisfazê-lo, porém, $Y$ teria que ir para $C$, o que pioraria a situação desta instituição.

3) As preferências responsivas permitem comparar quaisquer duas turmas? Dada a lista de alunos $W>X>Y>Z$, compare as seis possíveis turmas de dois alunos cada.

Resposta: Não há como comparar $\{W, Z\}$ com $\{X, Y\}$ (ROTH, 1985, p. 285). Para que a preferência seja responsiva, temos somente:

$$
\{W, X\}>\{W, Y\}>\{W, Z\} ?\{X, Y\}>\{X, Z\}>\{Y, Z\}
$$


4) Equipes sempre têm preferências responsivas?

Resposta: Não, porque uma empresa ou clube esportivo pode procurar, para sua equipe, profissionais que trabalhem melhor juntos, de modo que substituir um membro por outro mais qualificado ainda pode prejudicar o desempenho de seu parceiro e da equipe.

A teoria de alocação entre estudantes e universidades tem amplo interesse porque pode modelar também a formação de vínculo empregatício não só entre médicos residentes e hospitais, mas, em geral, entre trabalhadores e firmas. Contudo, é necessário incluir a questão de salários e, também, a hipótese de substitutabilidade que o exercício identifica: esse é o tema do Capítulo 6 de Roth; Sotomayor (1990).

\subsection{Casos reais: residência médica e escolas públicas}

A importância do algoritmo de Gale-Shapley e suas extensões é sua aplicação no mundo real. Um dos principais exemplos é a criação de um sistema de alocação de residentes em hospitais nos Estados Unidos, o NIMP (National Intern Matching Program), hoje chamado NRMP (National Resident Matching Program). Fazemos, aqui, uma apresentação do desenvolvimento desse sistema, baseada em Roth, Sotomayor (1990, p. 2-5), com detalhes extraídos dos artigos especializados Roth (1984) e Roth; Peranson (1999).

Depois, citamos alguns aspectos da alocação de estudantes em escolas públicas também em cidades americanas, com base nos artigos da premiação Nobel: Royal Swedish Academy of Sciences (2012a) e (2012b).

\section{O mercado da residência médica}

No começo do século XX, quando as residências em hospitais foram instituídas como forma de pós-graduação médica, existiam mais vagas que candidatos, de modo que o processo de escolha dos residentes pelos hospitais era muito competitivo. A primeira solução encontrada pelos hospitais nos Estados Unidos, a fim de obter os melhores estudantes, foi adiantar cada vez mais as propostas aos futuros residentes. 
Consequentemente, nos anos 40, um estudante já tinha de escolher onde faria sua residência dois anos antes de graduar-se (quando, então, faria residência). Entretanto, esta estratégia não era realmente favorável para nenhum dos lados, pois os hospitais contratavam seus futuros residentes sem ao menos saber suas notas finais (ou seja, o desempenho em aula de seus dois últimos anos de formação), enquanto os alunos escolhiam os hospitais e programas de residência sem terem terminado seus estudos, de modo que o processo de aprendizado em si ficava subordinado aos resultados dos programas de residência pelas propostas dos hospitais.

Com o propósito de evitar tal situação, ajustes foram feitos pela Association of American Medical Colleges (AAMC), os quais determinaram que as propostas não deveriam ser feitas antes do fim do penúltimo ano de graduação, o que solucionou a situação desfavorável de acordos precoces.

Não obstante, continuaram a ocorrer problemas nesse mercado, porque nem todos os candidatos rapidamente decidiam aceitar ou não as propostas, devido ao fato de esperarem receber propostas melhores ou serem chamados em alguma lista de espera em um hospital mais preferível. Assim, a segunda alteração empregada foi a diminuição do prazo que um futuro residente tinha para decidir se aceitava ou não uma proposta.

Dentro dessa nova estratégia, os prazos foram cada vez mais reduzidos, até o momento em que, em 1950, se decidiu por um prazo de somente 12 horas.

Essa solução não foi eficiente devido ao fato de que funcionava mais como um mecanismo de pressão por escolhas e, portanto, de frustação para ambos os lados, do que um mecanismo que realmente considerasse os anseios e o direito de escolha de cada agente. Dessa forma, a partir desse ano, começaram as discussões em favor da necessidade de implementação de um sistema centralizado para alocação de residentes e hospitais nos Estados Unidos. Decidiu-se primeiro pela adoção de um sistema centralizado de forma experimental. 
Esse sistema constituía-se da ordenação de listas de preferência que os estudantes elaboravam quanto aos hospitais a que eles mandavam suas propostas, ao passo que os hospitais também ordenavam os estudantes dos quais recebiam inscrições. Tais listas de preferência de ambos os grupos eram, então, submetidas a um escritório central, que determinava um emparelhamento com base em uma simulação de alocações prioritárias entre estudantes e hospitais ( $1^{\circ}$ com $1^{\circ}, 1^{\circ}$ com $2^{\circ}$ etc., não produzindo resultados estáveis).

A partir dos resultados experimentais do sistema centralizado, este começou a ocorrer de forma oficial; porém, a participação de estudantes e hospitais era voluntária. Entretanto, durante o experimento, o sistema já foi criticado pelos candidatos estudantes devido ao fato de promover alocações mais favoráveis aos interesses dos hospitais, em vista da ordem de alocação das prioridades, motivando os estudantes a serem "realistas" em suas preferências e identificar suas opções mais viáveis, não as mais desejadas, impondo-lhes um ônus contrapedagógico.

Assim, em 1951, esse algoritmo foi substituído por um novo, o NIMP, muito semelhante a Gale-Shapley, embora com os hospitais no que consideramos o papel de proponentes e os estudantes como seletores. Seu sucesso (medido pela longevidade do processo), mesmo em vista disso, deveu-se à produção de alocações estáveis. (Roth demonstrou que esse processo é mais complicado que o de Gale-Shapley, ainda que acabe produzindo a mesma alocação estável; veja Roth; Sotomayor, 1990, s. 5.4.)

Nas décadas seguintes, novos problemas apareceram. Um dos mais importantes, devido ao seu grande impacto no sistema, foi a situação conflituosa em que muitos casais de médicos não se inscreviam no sistema por desejarem estar próximos em suas alocações, o que tornava o processo instável em virtude do número crescente de estudantes (casais) e hospitais que faziam e aceitavam propostas por fora do sistema. Outro foi a dificuldade de hospitais em áreas rurais ou remotas obter residentes, imposta pelas preferências dos estudantes por grandes centros e inevitável, em vista da 
propriedade que destacamos na seção anterior (as vagas não preenchidas são sempre as mesmas em qualquer alocação estável).

Por isso, foi necessário incluir-se uma nova opção no sistema. Até 1982, os casais de futuros residentes puderam se inscrever de forma conjunta a partir de um "algoritmo de casais". Ambos os membros do casal enviavam suas listas de preferências ao sistema, porém um deles era escolhido, por eles, como "membro líder", de forma que fosse o primeiro a ser alocado pelo sistema. Seu parceiro, ao invés de ser emparelhado da mesma forma, tinha, então, sua lista de preferências editada, a fim de excluir lugares distantes da opção obtida pelo membro líder. Desta maneira, o segundo membro seria alocado à melhor possibilidade de programa de residência de um hospital na vizinhança da opção do parceiro "líder" no programa de alocação.

Porém, uma tal alocação era provavelmente instável, porque o líder poderia obter uma posição boa, enquanto o parceiro acabava em uma opção ruim, ditada pela localidade (não necessariamente uma de suas melhores opções naquela localidade, já eliminadas também), enquanto o líder e o parceiro poderiam ser preferidos por outra localidade e tal alocação, para o líder, fosse perfeitamente aceitável, e, não, uma piora significativa.

A partir de 1983, então, cada casal pôde indicar uma única lista de preferências de pares de hospitais. Roth e Sotomayor, entretanto, descobriram que um tal cenário pode não ter soluções estáveis; Ronn concluiu que o próprio problema é computacionalmente complexo. Para ver a instabilidade, note que, se um membro do casal é rejeitado no processo, ambos se movem (como casal) à sua próxima opção de pares de hospitais, ou seja, o outro membro desiste em essência da vaga que tinha e o hospital desta vaga fica a lamentar as rejeições que, por causa desse membro, fez a outros estudantes não significativamente piores.

Nos anos 90, mudanças no financiamento do setor de saúde nos EUA afetaram o mercado de trabalho médico e, então, o de residência médica, fortalecendo discussões sobre o sistema de alocação. Duas questões foram destacadas: o privilégio dos hospitais como 
grupo proponente e a possibilidade de os estudantes necessitarem estratégias "realistas" como grupo seletor. Para evitar um novo descrédito do programa central e a consequente desorganização crítica do mercado, propôs-se uma revisão do algoritmo. Roth e Elliott Peranson elaboraram o novo procedimento, que entrou em funcionamento em 1998.

O novo algoritmo é descrito por Roth; Peranson (1999, p. 756757) e baseia-se em um algoritmo de Roth e Vande Vate para eliminar instabilidades uma a uma: muito resumidamente, trata-se de colocar novos candidatos em um conjunto de agentes proponentes, um por vez; realizar sua proposta a um hospital, que pode desencadear uma cadeia de propostas e rejeições pelos demais agentes como em Gale-Shapley e, também, rejeições extras de ou por casais de médicos ou pares de programas associados para $1^{\circ}$ e $2^{\circ}$ anos de residência; essas rejeições extras são armazenadas temporariamente e dirimidas após a cadeia principal.

Mesmo com a mudança para estudantes como grupo proponente e o tratamento homogêneo de casais, Roth e Peranson constataram que as alocações obtidas por este método eram pouquíssimo diferentes daquelas pelo método anterior, então oficial, utilizando-se os dados de alocação de cada ano. A esse respeito, resumimos o último parágrafo da Seção A (pág. 761) e sua nota de rodapé: apesar da diferença entre as alocações ótimas para hospitais e para estudantes diferirem em poucos agentes, para estes, a diferença é substancial; por um lado, um hospital (ou universidade em geral) pode não ver diferença entre seus candidatos classificados até mesmo várias posições em separado, enquanto um estudante pode não ter uma distinção clara entre suas primeiras duas opções, mas terá, com certeza, entre as várias opções, por sua localização em cidades distantes etc. (Os extremos ótimos serem tão próximos indica o pouco número de alocações estáveis relativo ao tamanho grande do mercado, o que é explicado na p. 768 como resultado de cada agente poder entrevistar-se com e avaliar poucas opções, em vista dos custos de viagem, tempo e administração.) 


\section{As matrículas nas escolas públicas}

Dificuldades na matrícula de alunos em escolas públicas são uma ocorrência global. Até o começo do século XXI, os sistemas de alocação em várias cidades americanas sofriam problemas, enquanto tentavam alocar os alunos diretamente a suas primeiras opções de escola. Como se verificava em New York e Boston, a melhor estratégia do estudante era ser "realista".

Na cidade de New York, os alunos tinham um número limitado de opções de escola para indicar (cinco opções), enquanto as escolas decidiam quem aceitar, rejeitar ou pôr em espera também em um número limitado de rodadas (três rodadas), enquanto alunos e vagas não alocados nesse processo eram resolvidos posteriormente por um escritório. Desse modo, a tendência das escolas era selecionar os alunos que as indicassem em primeiro lugar, mas com o agravante de congestão. Mais especificamente, quantidades significativas de alunos eram matriculadas em escolas pelas quais não indicavam nenhuma preferência, enquanto as escolas não podiam considerar alunos suficientes durante sua seleção.

Em Boston, um procedimento centralizado já era usado desde a primeira etapa, porém tentando justamente matricular os alunos em suas primeiras opções, depois em suas segundas opções e assim sucessivamente.

Nota dos Autores: Podemos observar que a tendência em alocar candidatos às melhores preferências como um "princípio da prioridade" (ou "aceitação imediata"), se não embutida já no design do processo, será de qualquer modo muito natural para os agentes seletores (por comodidade, narcisismo ou receio de perder o proponente), ou mesmo para o planejador central (por intuição e por baixo custo computacional). Contudo, esse princípio parece conduzir mais rapidamente à instabilidade: cf. o histórico acima e também Roth; Sotomayor (1990, s. 5.5.1.1) a respeito da história da alocação de residência médica no Reino Unido. 
Convém destacar que as preferências das escolas (ou da administração central, isto é, do sistema como um todo) pelos alunos podem não ser explicitamente formuladas ou considerar somente desempenho em notas e frequência, mas acomodar outras considerações, como proximidade da escola à residência do aluno ou irmãos do candidato que já estudam na escola.

Alvin Roth e seus colegas trabalharam nos sistemas de New York e Boston a partir de 2003, implementando programas de matrícula baseados em Gale-Shapley com propostas realizadas pelos alunos, reduzindo sua necessidade de procurar por estratégias. Esses trabalhos envolvem, também, a adequação dos programas aos regulamentos ou normas de cada sistema. 